\title{
Особенности биоэлектрической активности головного мозга в зависимости от степени тяжести бронхиальной астмы
}

ГУ "Дальневосточный научный центр физиологии и патологии дыхания Сибирского отделения РАМН": 675000, Амурская обл., Благовещенск, ул. Калинина, 22

\section{E.V.Ermakova, Yu.M.Perelman, V.P.Kolosov \\ Peculiarities of brain bioelectric activity depending on severity of bronchial asthma}

\begin{abstract}
Summary
Peculiarities of brain bioelectric activity were analyzed in 102 patients with exacerbation of bronchial asthma of different severity. The absolute majority of patients had abnormal functional status of the central nervous system with diffuse changes of brain bioelectric activity of different intensity in electroencephalogram. As the severity of bronchial asthma grew, the midbrain stem structures of brain became involved. Key words: electroencephalography, power spectrum of cerebral bioelectrical activity, bronchial asthma, severity of disease.
\end{abstract}

\section{Резюме}

У 102 больных бронхиальной астмой (БА) с различной степенью тяжести заболевания в период обострения проанализированы особенности биоэлектрической активности головного мозга. У абсолютного большинства больных выявлены нарушения функционального состояния центральной нервной системы, проявляющиеся на электроэнцефалограмме диффузными изменениями биоэлектрической активности головного мозга различной степени выраженности. По мере нарастания степени тяжести БА отмечается вовлечение срединно-стволовых структур мозга.

Ключевые слова: электроэнцефалография, спектр мощности биоэлектрической активности мозга, бронхиальная астма, степень тяжести.

Сложный патогенез любых форм бронхиальной астмы (БА) не ограничивается только реакциями аллергического воспаления, а включает в себя ряд механизмов 2-го порядка, в частности нейрогенных [1, 2]. Роль нейрогенных механизмов в поддержании хронического воспаления дыхательных путей и патогенезе БА все еще остается неясной [3]. Поскольку изменения в тонусе мускулатуры бронхов при БА происходят быстро, давно было сделано предположение, что при этом заболевании может иметь место расстройство регуляции вегетативной нервной системы, проявляющееся дисбалансом между возбуждающим и тормозящим влиянием и приводящее к чрезмерной "судорожности" дыхательных путей. Известно немного исследований, посвященных анализу данных электроэнцефалографии у больных БА, отражающих текущее функциональное состояние головного мозга. Поскольку методы спектрального анализа электроэнцефалограмм (ЭЭГ) показали высокую информативность при различной неврологической патологии [4], представляется оправданным их использование для оценки возможного участия центральных механизмов регуляции в формировании патологического паттерна бронхомоторного тонуса у больных БА с различной степенью тяжести заболевания.

Целью настоящей работы явилось изучение особенностей биоэлектрической активности коры головного мозга у больных БА различной степени тяжести в период обострения.

\section{Материалы и методы}

В исследовании участвовали 102 пациента со смешанной формой БА в фазе обострения в возрасте от 18 до 50 лет (56 женщин и 46 мужчин). Средний возраст больных составил $34,5 \pm 11,1$ года. Степень тяжести БА определяли в соответствии с классификацией, принятой Международным соглашением [5].

Пациенты были разделены на следующие группы: 1-я группа - 59 больных БА легкой степени, средний возраст $-31,4 \pm 10,7$ года; 2-я группа -25 больных БА средней степени тяжести, средний возраст $-37,3 \pm 10,0$ года; 3-я группа - 18 пациентов с БА тяжелой степени, средний возраст - 42,2 \pm 5,4 года. Контрольную группу составили 30 практически здоровых лиц - 11 мужчин и 19 женщин, средний возраст $-33,5 \pm 11,9$ года.

Комплексное обследование осуществлялось при поступлении пациентов в стационар до назначения стандартной базисной терапии. Вентиляционную функцию легких оценивали по данным спирометрии форсированного выдоха с анализом кривой потокобъем на аппарате Ultrascreen (Erich Jaeger, Германия).

Электроэнцефалографическое исследование проводили с помощью компьютерного анализатора электрической активности мозга ЭЭГА-21/26 "Энцефалан-131-03" (Россия), размещая электроды на поверхности головного мозга в соответствии с между- 
народной схемой отведений "10-20". Проводилась визуальная оценка ЭЭГ. Методом автоматизированного количественного частотного анализа ЭЭГ оценивалась мощность спектра по частотным диапазонам в процентах для $\alpha-, \beta-, \theta-, \delta$-активности в обоих полушариях.

Статистический анализ полученного материала проводили с помощью экспертной системы "Автоматизированная система для научных исследований в области физиологии и патологии дыхания человека" на основе стандартных методов вариационной статистики с оценкой достоверности различий по t-критерию Стьюдента. Анализ распространенности признака в сравниваемых группах (частота альтернативного распределения) проводили по $\chi^{2}$-критерию Пирсона для 4-польной таблицы. Значение полученного критерия $\chi^{2}$ сравнивали с граничными значениями: 3,84 для 5\%-й вероятности и 6,63 для 1\%-й вероятности нулевой гипотезы.

\section{Результаты и обсуждение}

По результатам оценки вентиляционной функции легких во всех группах больных БА, по сравнению с контрольной, достоверно снижались скоростные показатели форсированного выдоха.

Проведенный анализ фоновой записи ЭЭГ у больных БА показал, что у подавляющего большинства пациентов выявлялись диффузные изменения биоэлектрической активности головного мозга различной степени выраженности (рис. 1). В группе пациентов с легким течением БА в $17 \%$ регистрировался вариант условно нормальной ЭЭГ, в то время как у пациентов со средним и тяжелым течением заболевания вариант нормальной ЭЭГ не встречался.

Легкие диффузные изменения биоэлектрической активности головного мозга были выявлены у $41 \%$ больных БА с легким течением заболевания, у $36 \%-$ со среднетяжелым и у $28 \%$ - с тяжелым течением БА. Гораздо чаще регистрировались умеренные общемозговые изменения, выявляемые больше у пациентов со средним и тяжелым течением заболевания. У подавляющего большинства обследуемых была об-

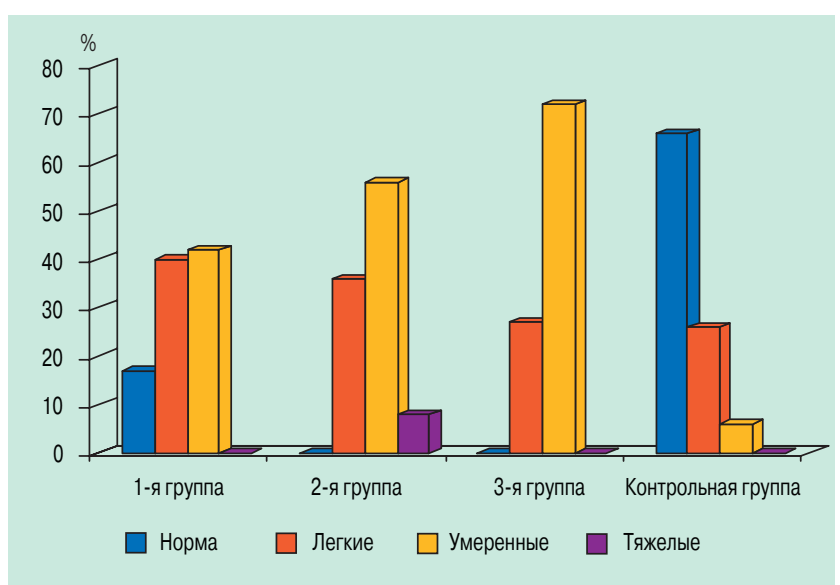

Рис. 1. Частота встречаемости диффузных поражений биоэлектрической активности головного мозга у больных БА наружена дисфункция срединных структур головного мозга. Так, у больных БА легкого течения дисфункция срединных структур головного мозга гипоталамического уровня регистрировалась в $16 \%$ случаев, диэнцефального уровня - в $42 \%$, мезенцефального уровня - в $16 \%$. В группе пациентов со среднетяжелым течением БА на фоне легких диффузных изменений биоэлектрической активности головного мозга в 55 \% случаев имелись признаки дисфункции срединно-стволовых структур на уровне гипоталамуса и у $11 \%$ - на уровне диэнцефальных структур.

Умеренные диффузные изменения биоэлектрической активности головного мозга были выявлены у $56 \%$ больных среднетяжелой БА, из них у $71 \%$ отмечались признаки поражения срединных структур мозга диэнцефального уровня и у $21 \%$ - мезенцефального уровня. У 8 \% обследованых со среднетяжелым течением БА были выражены диффузные изменения биоэлектрической активности головного мозга, которые были грубыми, эпилептиформными, с признаками вовлечения неспецифических срединных структур мозга диэнцефально-мезенцефального уровня.

Исследование показателей ЭЭГ в группе больных БА тяжелого течения выявило у 28 \% легкие диффузные изменения биоэлектрической активности головного мозга, из них у $40 \%$ - дисфункцию срединно-стволовых структур мозга на гипоталамическом уровне и у $40 \%$ - на диэнцефальном уровне. В $72 \%$ случаев регистрировались умеренные общемозговые изменения биоэлектрической активности, из них признаки дисфункции срединно-стволовых структур мозга на гипоталамическом уровне встречались у $61 \%$, на диэнцефальном уровне - у $38 \%$ больных.

К настоящему времени в многочисленных работах показано, что нарушение функционального состояния центральной нервной системы (ЦНС) может влиять на развитие заболевания, т. к. именно центральные регуляторные механизмы обеспечивают настройку всех систем, подготавливают и определяют реакцию организма в процессе заболевания. Обнаруженные нами диффузные изменения биоэлектрической активности и участие стволовых структур головного мозга различного уровня указывают на нарушение механизмов адаптации у больных БА.

Проведенное топографическое картирование спектральной мощности основных ритмов ЭЭГ с большей точностью, чем при визуальном анализе, позволило оценить изменения биоэлектрической активности головного мозга при БА.

В группах больных БА легкой и средней тяжести, в сравнении с контрольной, достоверных различий со стороны индекса мощности $\alpha$-ритма выявлено не было. Однако у пациентов с тяжелым течением БА мощность $\alpha$-диапазона была достоверно выше во всех отведениях головного мозга, в сравнении с контрольной группой. Аналогичные изменения выявлены и в сравнении показателей больных 3-й и 1-й групп: наиболее выраженные различия мощности $\alpha$-ритма отмечались в теменно-затылочной, центральной, 


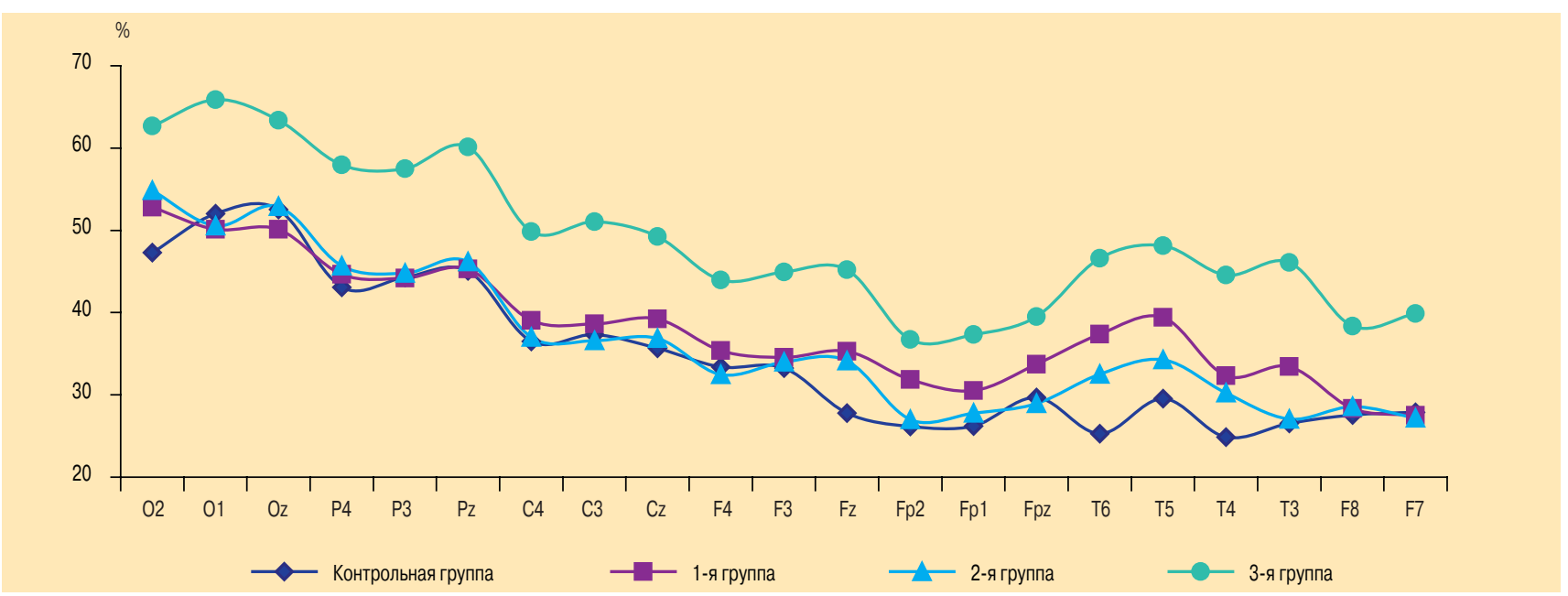

Рис. 2. Изменение индекса мощности $\alpha$-ритма

средне- и передне-височной областях мозга с 2 сторон (рис. 2).

Поскольку для $\alpha$-ритма характерны свое пространственное распределение и преимущественная локализация, усиление $\alpha$-активности у больных с тяжелым течением БА указывает на подавление деятельности ретикулярной формации ствола, заднего гипоталамуса, неспецифических ядер таламуса и хвостатого ядра и доминирование восходящих влияний на кору со стороны переднего гипоталамуса, активность которого, по данным литературы, коррелирует с признаками возбуждения парасимпатической нервной системы [6].

Дальнейший анализ спектра мощности основных ритмов ЭЭГ показал достоверное различие индекса мошности $\beta$-ритма при сравнении как групп больных БА между собой, так и с контрольной группой (рис. 3).

Выявленные изменения носили разнонаправленный характер. Так, индекс мощности $\beta$-ритма был самым высоким в группе пациентов со среднетяже- лым течением БА, несколько снижался в группе пациентов с легким течением заболевания и резко снижался в группе пациентов с тяжелым течением болезни. Значения индекса мощности $\beta$-диапазона в 1-й и 2-й группах не отличались от контрольной группы, однако в 3-й группе, по сравнению с контрольной, были выявлены достоверные различия. Достоверны различия показателей в затылочной, теменной, центральной областях мозга, височной области левого полушария и задне- и средне-височной областях правого полушария.

У пациентов 2-й группы, в отличие от 1-й и 3-й групп, была отмечена достоверно более высокая относительная мощность $\beta$-активности в целом по всем отведениям, но наиболее достоверными были различия показателей в задне- и средне-височной областях левого полушария, в центральной и средневисочной областях правого полушария (по сравнению с 1-й группой). Также выявлены достоверные различия индекса мощности $\beta$-ритма в сторону его увеличения у пациентов 1-й группы, в отличие от 3-й

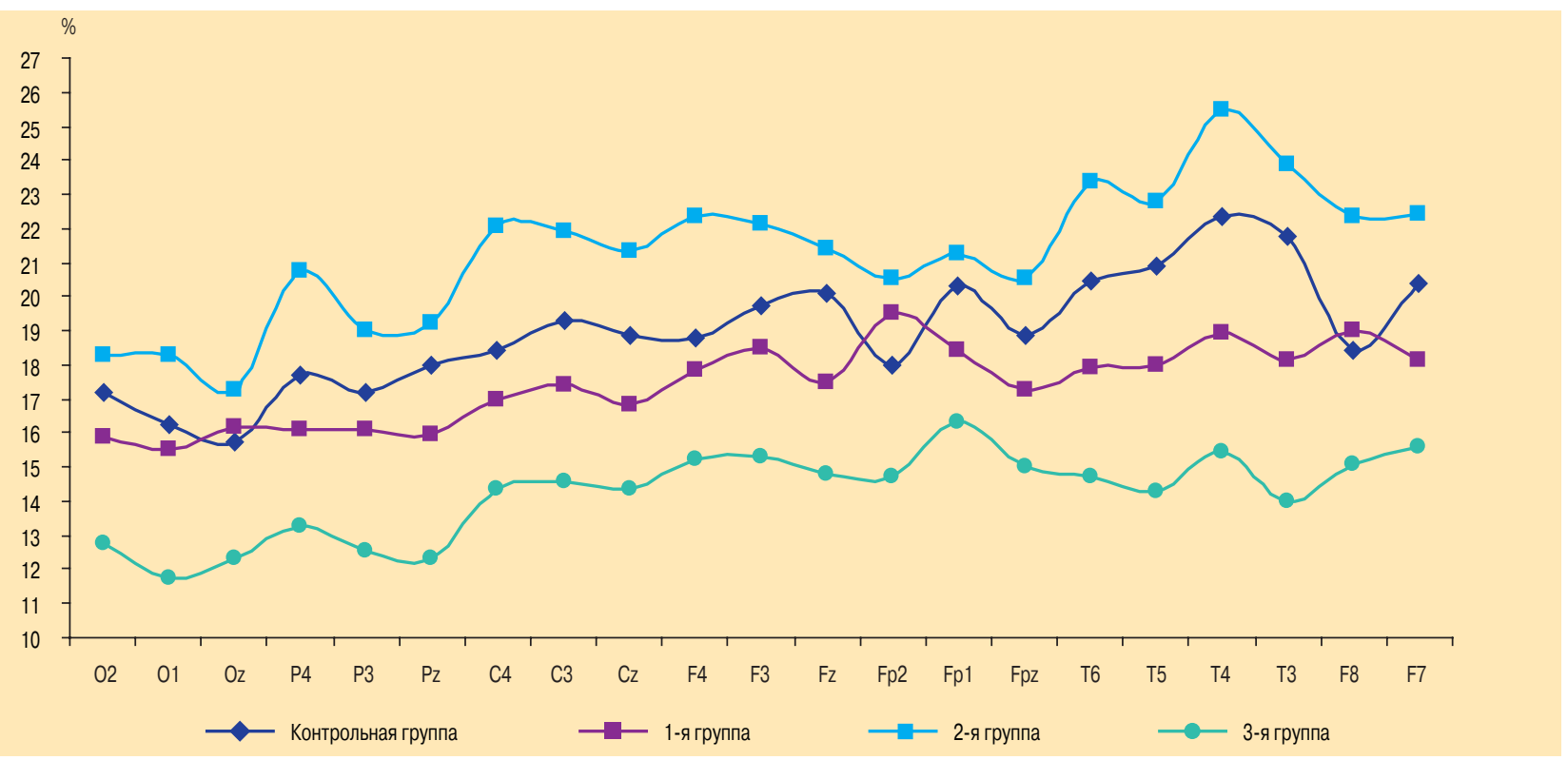

Рис. 3. Изменение индекса мощности $\beta$-ритма 


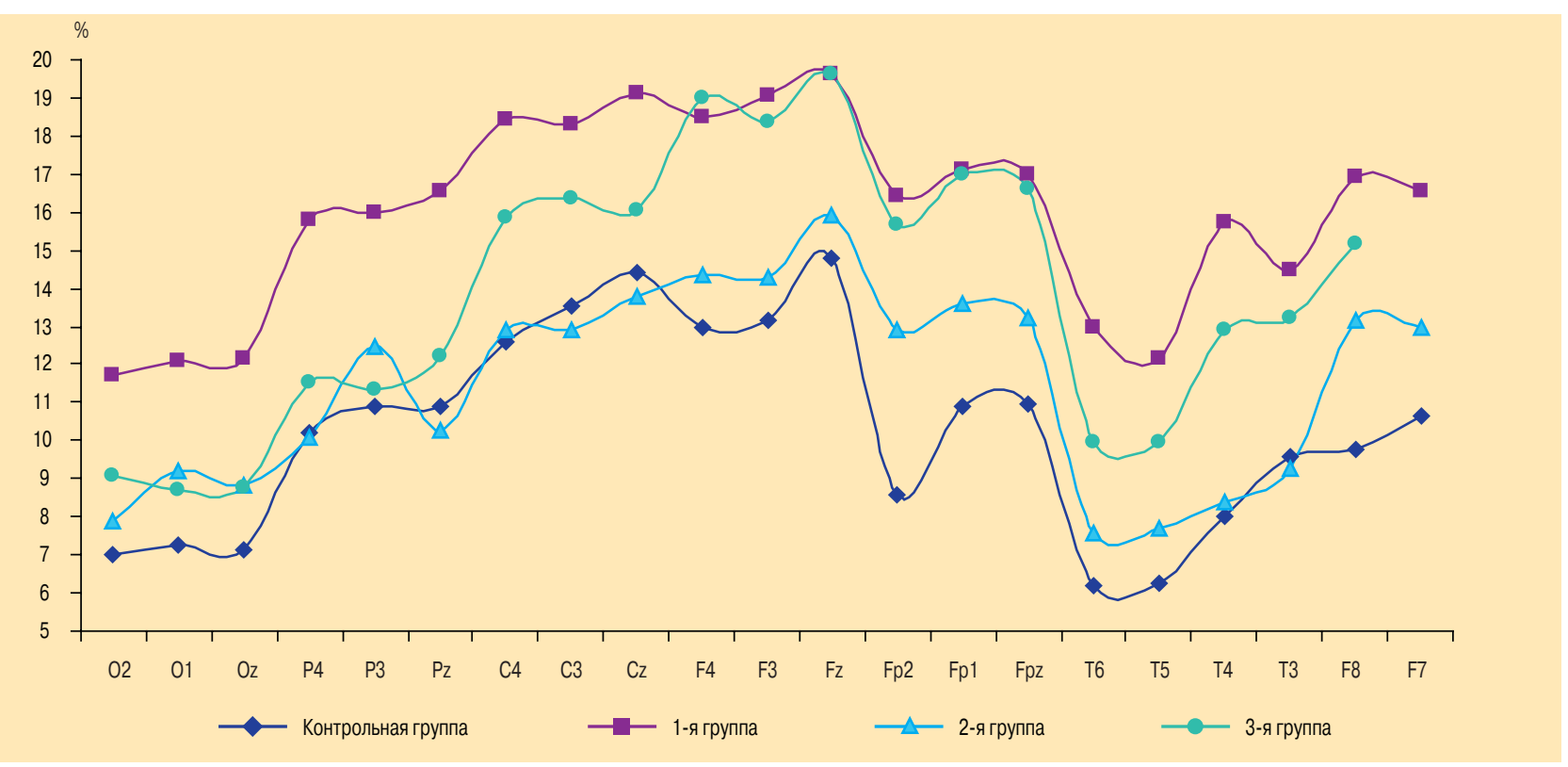

Рис. 4. Изменение индекса мощности $\theta$-ритма

группы, с акцентом в лобной области правого полушария, задне- и средне-височной областях левого полушария.

При анализе относительного значения мощности активности $\beta$-диапазона наиболее значимыми были различия между 2-й и 3-й группами. Так, уровень мощности $\beta$-ритма был значительно ниже у пациентов 3-й группы, достигая степени статистической достоверности во всех отведениях головного мозга.

По наблюдениям А.Я.Могилевского [7], воздействие на кору головного мозга со стороны ретикулярной формации ствола происходит в основном в диапазоне колебаний $\alpha$-активности. Следовательно, увеличение спектра мощности $\alpha$-ритма в группе пациентов с БА средней тяжести можно расценить как возбуждение активирующих ретикулокортикальных систем, отражающих высокий уровень функциональной активности мозга. У пациентов с легким и тяжелым течением БА обнаруженные изменения, напротив, можно рассматривать как снижение уровня функциональной активности ретикулокортикальных систем, сопровождающееся сокращением афферентного притока и усилением зависимости нейронной активности мозга от эндогенных механизмов [8].

Индекс мощности активности $\theta$-диапазона был выше у пациентов с легкой и тяжелой БА во всех отведениях, в сравнении с показателями контрольной группы, и незначительно отличался в группе пациентов со среднетяжелым течением заболевания (рис. 4). Повышение мощности $\theta$-активности, регистрировавшееся во всех отведениях, было достоверно более выражено у пациентов 1-й группы, в отличие от здоровых лиц. Во 2-й группе эти различия были достоверны лишь в лобной и передне-височной областях правого полушария. В 3-й группе достоверные различия индекса мощности $\theta$-активности выявлены в лобных и височных областях как правого, так и левого полушарий.

Имеющиеся в литературе данные указывают на связь $\theta$-активности у человека в условиях патологии

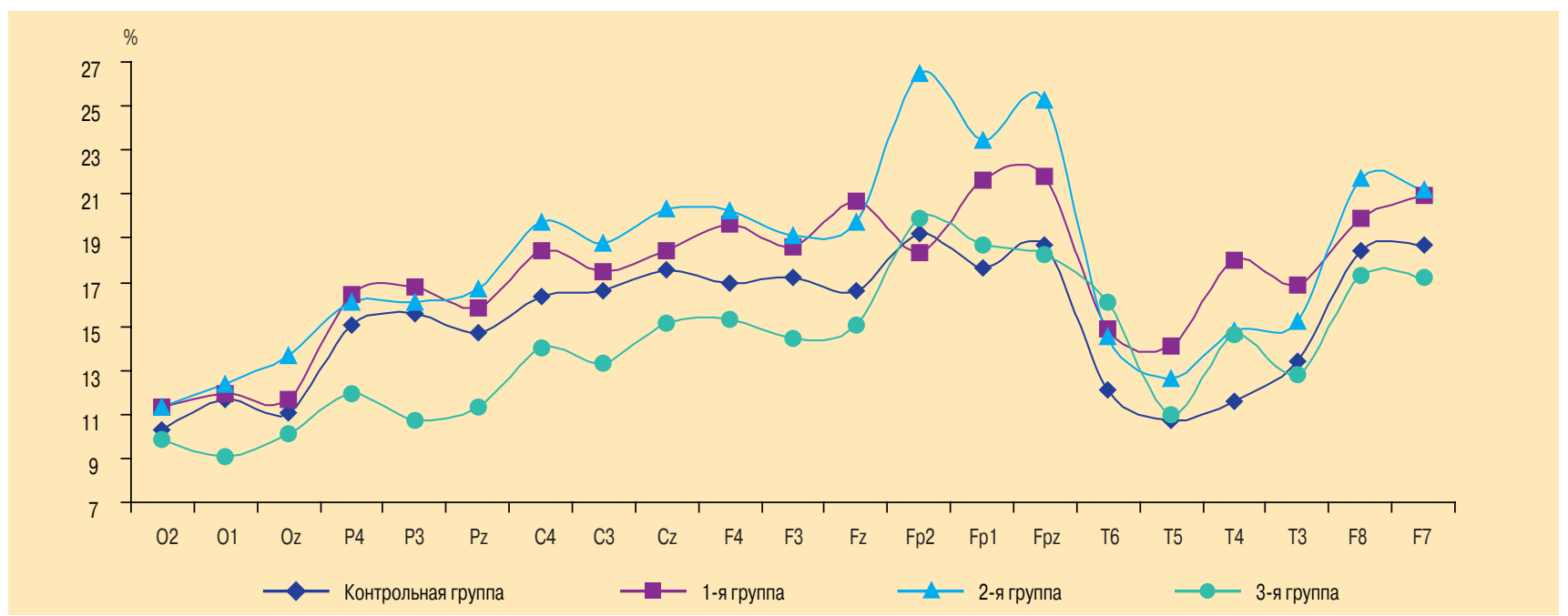

Рис. 5. Изменение индекса мощности $\delta$-ритма 
или с непосредственным поражением коры больших полушарий, или с влиянием со стороны лимбических структур, главным образом гипокампа, а диффузно регистрируемая на ЭЭГ $\theta$-активность свидетельствует о поражении диэнцефальной области.

При анализе картограмм больных БА обнаружены некоторые изменения со стороны спектральной мощности $\delta$-диапазона, отражающего участие верхнестволовых, более глубоких, структур мезенцефального уровня (рис. 5.) Индекс мощности $\delta$-колебаний был выше в группах больных БА легкого и среднетяжелого течения, по сравнению с контрольной группой, но наиболее достоверные различия относительного значения мощности $\delta$-ритма получены при сравнительном анализе групп между собой. Во 2-й группе, по сравнению с 3-й, значение мощности $\delta$-ритма было достоверно выше в теменной области левого полушария, в центральной и лобной областях с 2 сторон. Также были отмечены различия в лобной области справа при сравнении 1-й и 2-й групп в сторону увеличения мощности $\delta$-ритма во 2-й группе.

По данным литературы, факторами, вызывающими изменения деятельности корковых нейронов с появлением $\delta$-активности, являются главным образом гипоксия, нарушения метаболизма и дисциркуляторные расстройства в системах ликворообращения и кровообращения, а под действием дистантных влияний возникновение $\delta$-активности в лобных областях мозга указывает на поражение оральных отделов ствола мозга [6].

\section{Заключение}

Таким образом, у абсолютного большинства больных БА (> 80 \%) отмечаются нарушения функционального состояния ЦНС, проявляющиеся на ЭЭГ диффузными изменениями биоэлектрической активности головного мозга различной степени выраженности. По мере нарастания тяжести БА отмечается вовлечение срединно-стволовых структур мозга, и уровень вовлекаемых структур прямо про- порционален степени тяжести заболевания, что, в свою очередь, указывает на важную патогенетическую роль изменений механизмов центральной регуляции в формировании нейрореспираторных нарушений у больных БА и может способствовать модификации подходов к определению лечебной тактики.

\section{Литература}

1. Булатов П.К., Федосеев Г.Б. Бронхиальная астма. Л.: Медицина: 1975.

2. Butler C.A., Heaney L.G. Neurogenic inflammation and asthma. Inflamm. Allergy Drug Targets 2007; 6 (2): 127-132.

3. Groneberg D.A., Quarcoo D., Frossard N., Fisher A. Neurogenic mechanisms in bronchial inflammatory diseases. Allergy 2004; 59 (11): 1139-1152.

4. Зенков Л.Р., Ронкин М.А. Функциональная диагностика нервных болезней. М.: МЕДпресс-информ; 2004.

5. Global initiative for asthma. Global strategy for asthma management and prevention [Electronic data]. National Institute of Health National Heart, Lung and Blood Institute. Revised 2006. Available at: http://www.ginasthma. com.

6. Жирмунская Е.А. Клиническая электроэнцефалография. М.: МЭЙБИ; 1991.

7. Могилевский А.Я. Влияние стимуляции заднего гипоталамуса на ЭЭГ неокортекса. Журн. высш. нерв. деят. 1971; 21 (6): 1268-1278.

8. Зенков Л.Р. Клиническая электроэнцефалография с элементами эпилептологии. М.: МЕДпресс-информ, 2002. 17-39.

\section{Информация об авторах}

Ермакова Елена Владимировна - к. м. н., научный сотрудник лаборатории функциональных методов исследования дыхательной системы ГУ "Дальневосточный научный центр физиологии и патологии дыхания СО РАМН"; тел.: (4162) 44-12-27; e-mail: elena-ryltseva@rambler.ru

Перельман Юлий Михайлович - д. М. н., проф., зам. директора по научной работе ГУ "Дальневосточный научный центр физиологии и патологии дыхания СО РАМН"; тел.: (4162) 44-12-27; e-mail: jperelman @mail.ru

Колосов Виктор Павлович - д. м. н., проф., директор Гу "Дальневосточный научный центр физиологии и патологии дыхания СО РАMН"; тел.: (4162) 53-35-45; e-mail: kolosov@amur.ru 